\title{
Acute Bowel Obstruction After Hepaticojejunostomy Caused by Blood Clots
}

\author{
Mihir M. Shah, MD, Assar A. Rather, MD, Farrukh A. Khan, MD \\ General Surgery, Cleveland Clinic Foundation, Cleveland, OH, USA (Dr. Shah). \\ General Surgery, Bayhealth General Surgery, Dover, DE, USA (Dr. Rather). \\ General Surgery, Mercy Catholic Medical Center, Philadelphia, PA, USA (Dr. Khan).
}

\begin{abstract}
Introduction: We report a case of intestinal obstruction secondary to intramural blood clots at the level of the jejunojejunostomy anastomosis, causing an acute elevation of bilirubin levels.

Case Description: A 71-year-old woman who had undergone cholecystectomy and Roux-en-Y hepaticojejunostomy presented to the emergency department with severe abdominal pain 1 day after discharge. She was afebrile and her vital signs were stable. Her abdomen was distended but soft. Her liver function test results were normal at the time of discharge. However, her total bilirubin was noted to be $2.3 \mathrm{mg} / \mathrm{dL}$ at the time of readmission, 1 week after the original reconstruction. Eight hours later, her total bilirubin increased to $8.5 \mathrm{mg} / \mathrm{dL}$. A computed tomography (CT) scan revealed no bile leak but showed gross dilatation of the Roux limb and proximal small bowel and a collapsed distal small bowel. Re-exploration confirmed the CT scan findings. The proximal small-bowel limb was evacuated of the clots and the Roux limb decompressed. A CT scan obtained 4 weeks later showed resolution of the dilatation of the Roux limb and the proximal small bowel.

Discussion: Obstruction resulting from intramural blood clot formation in the small intestine is caused by inadequate hemostasis at the stapled anastomosis. Operative revision is rarely required. However, our patient had significant dilatation of the Roux limb and the small bowel, with a rapid elevation of serum bilirubin that required surgical exploration. Usually the cause is bleeding from the jejunojejunostomy, which may require reoperation and/or revision of the anastomosis.
\end{abstract}

Key Words: Intramural blood clots, Roux limb obstruction, Roux-en-Y hepaticojejunostomy, Bilirubin, Jejunojejunostomy.

Citation Shah MM, Rather AA, Khan FA. Acute bowel obstruction after hepaticojejunostomy caused by blood clots. CRSLS e2014.00188. DOI 10.4293/CRSLS. 2014.00188.

Copyright (C) 2014 SLS This is an open-access article distributed under the terms of the Creative Commons Attribution-Noncommercial-ShareAlike 3.0 Unported license, which permits unrestricted noncommercial use, distribution, and reproduction in any medium, provided the original author and source are credited.

Address correspondence to: Mihir M. Shah, MD, Cleveland Clinic Foundation (General Surgery), 9500 Euclid Avenue, A 100, Cleveland, OH 44195. Telephone: (215) 200-6256, Fax: (216) 445-7653, E-mail: ShahM@ccf.org

\section{INTRODUCTION}

In this case report, our patient presented with intestinal obstruction secondary to intramural blood clots at the level of the jejunojejunostomy anastomosis. The patient was 71 years old with a past medical history of myocardial infarction, hypertension, and hepatitis C. She had a hepaticojejunostomy performed as a result of injury to the common hepatic duct and left hepatic duct during laparoscopic cholecystectomy 1 week before this presentation.

\section{CASE REPORT}

A 71-year-old woman who had undergone cholecystectomy and Roux-en-Y hepaticojejunostomy presented to the emergency department with the chief complaints of severe abdominal pain and nausea 1 day after discharge (postoperative day 8). She was afebrile and her vital signs were stable. On physical examination, her abdomen was noted to be distended, soft, and tender around the incision; auscultation revealed normal bowel sounds. She was taking $81 \mathrm{mg}$ of aspirin daily. 
She had sustained an E3 hilar injury during laparoscopic cholecystectomy, for which she had undergone repair with a Roux-en-Y hepaticojejunostomy in a standardized fashion. A GIA $75-\mathrm{mm}$ stapler was used to fashion the jejunojejunostomy, with no evidence of bleeding. Her liver function test results were normal at the time of discharge. However, her total bilirubin was noted to be $2.3 \mathrm{mg} / \mathrm{dL}$ at the time of readmission. Eight hours after readmission, her total bilirubin had increased to 8.5 $\mathrm{mg} / \mathrm{dL}$. Computed tomography (CT) scan revealed gross dilatation of the Roux limb and the biliary limb and a collapsed distal small bowel (Figures 1 and 2). A decision was made to re-explore the area, and the patient was taken to the operating room after giving her informed consent.

Exploration of the abdominal cavity revealed marked dilatation of the biliary limb of the jejunum and Roux limb compared with the distal bowel, with intact biliary-enteric anastomosis. A small, 2.5-cm, side-to-side neojejunojejunostomy was performed using the proximal Roux limb and the distal small bowel (common channel) in relation to the original anastomosis (jejunojejunostomy), which was left untouched. At this time, the biliary limb was evacuated of the clots, and the Roux limb was also decompressed. An intraoperative cholangiogram was performed, which revealed good flow of the contrast in the biliary system (Figure 3).

At pathologic examination, the evacuated blood clots were organized and admixed with fibrin, vegetable material, and bacterial colonies. The patient recovered postoperatively and was discharged home with normal serum bilirubin

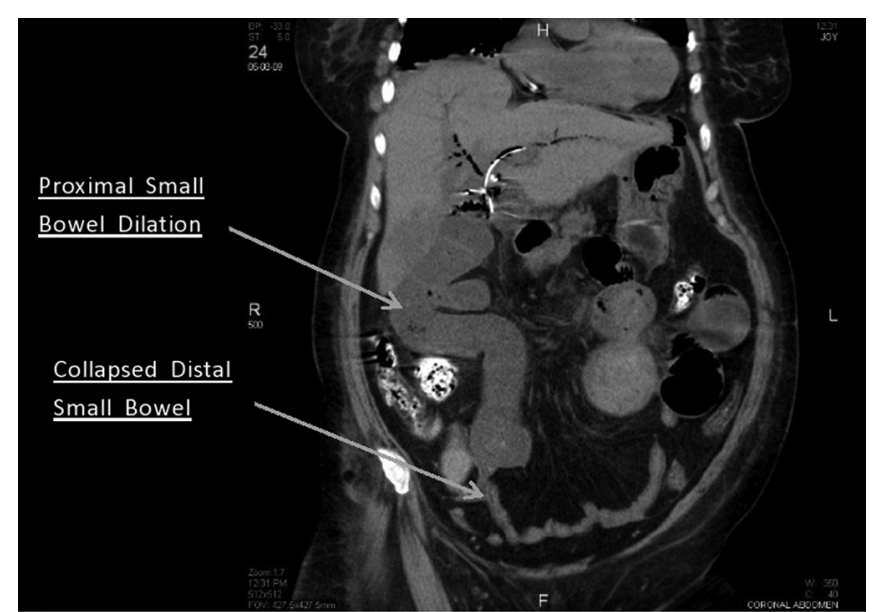

Figure 1. A CT scan showing gross dilatation of the Roux limb and proximal small bowel and a collapsed distal small bowel.

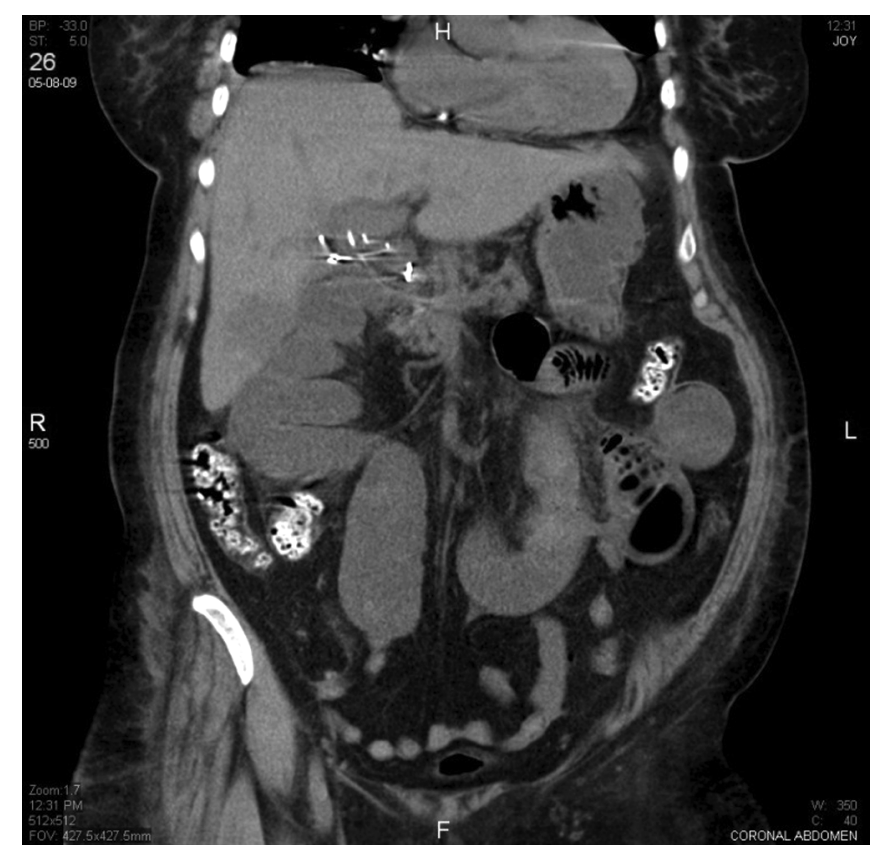

Figure 2. A CT scan showing a transition point in the right lower quadrant.

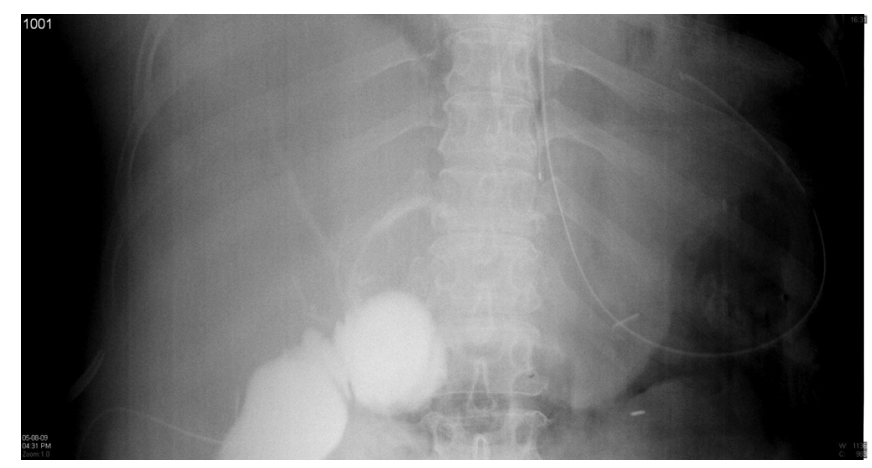

Figure 3. An intraoperative cholangiogram revealing patency of the biliary system and enhancement of the Roux limb.

levels. A CT scan obtained 4 weeks later showed resolution of the dilatation of the Roux limb and the proximal small bowel.

\section{DISCUSSION}

Roux described a bypass procedure for gastric outlet obstruction more than 115 years ago. ${ }^{1}$ Roux-en-Y reconstruction has been extensively used after gastrectomy for cancer, gastric bypass for obesity, and in hepaticojejunostomy or choledochojejunostomy as an end-to-side anastomosis.

Early small intestine obstruction has been well documented after laparoscopic gastric bypass surgery, with an incidence of $1.2 \%$. The average time of presentation 
is 15 days (range, 5-27). The different etiologies described are dietary noncompliance (46\%), anastomotic edema (23\%), narrowing of the jejunojejunostomy (23\%), and luminal clots (8\%). ${ }^{2}$ The complication rates for Roux-en-Y hepaticojejunostomy are relatively low in large series. ${ }^{3,4}$ Bowel obstruction (adhesion and intussusception) account for $4.5 \%$ of the complications; approximately $4 \%$ of the patients report pancreatitis or ascending cholangitis. ${ }^{4}$ The principles of technique that minimize the chances of complications are (1) the anastomosis must start by being as large as possible; (2) there must be complete apposition of the bile duct lining to the jejunal mucosa; and (3) there must be no tension. ${ }^{5}$

Small-bowel obstruction (SBO) caused by intramural hematoma is well described in the literature, ${ }^{6}$ but this case is unique because the hematoma, in addition to the $\mathrm{SBO}$, caused an acute elevation of bilirubin. The use of CT scan for patients with this clinical presentation has been a helpful diagnostic tool. ${ }^{7}$ Fresh blood appears as an area of increased density on a CT scan, with the density diminishing as the hematoma ages. ${ }^{8}$ Inappropriate use of a surgical stapler may result in complications including bleeding, leakage, and stenosis.9,10 Although the frequency of hemorrhage is low, ${ }^{11}$ surgeons must be careful in achieving adequate hemostasis.

The obstructive complications discussed here with the Roux-en-Y anastomosis are most likely to occur in the early postoperative period $(<3 \mathrm{mo}) .^{12}$ Obstruction resulting from intramural blood clot formation in the small intestine is caused by inadequate hemostasis at the stapled anastomosis and is rarely seen in the immediate postoperative period. ${ }^{12}$ In general, a hematoma undergoes spontaneous reabsorption in 14 to 90 days, ${ }^{13}$ making surgical intervention seldom necessary. Hence the treatment for intramural hematoma is nonoperative, ${ }^{13}$ and because most of these hematomas resolve spontaneously, ${ }^{13,14}$ surgical management is infrequently required.

In our patient, it appears the original surgeon noted that the gall bladder was attached to the common bile duct with no cystic duct. It was not reported to be particularly difficult technically, but bile leakage from the common bile duct was noted. Hence we were consulted intraoperatively for management of the bile duct injury. Postoperatively, our patient had significant dilatation of the Roux limb and the biliary limb of the jejunum, with a rapid elevation of serum bilirubin. This obstructive bilirubinemia required surgical exploration, including an intraoperative cholangiogram at the end of the procedure, to rule out other causes of obstructive jaundice and ensure patency of the biliary system.

\section{CONCLUSION}

Intramural blood clot formation causing $\mathrm{SBO}$ should be included in the differential diagnosis when confronting similar clinical findings in the immediate postoperative period after a hepaticojejunostomy. Altered anatomy may present a diagnostic challenge because the presentation and imaging modalities may be vague and inconclusive. ${ }^{15} \mathrm{~A}$ high index of suspicion is pivotal to prompt early diagnosis and expeditious surgical intervention, to relieve the bowel obstruction and the bile duct obstruction. The management of each patient should be individualized based on the clinical condition, laboratory data, and imaging findings. Aspirin taken perioperatively in high-risk cardiac patients has been shown to decrease major adverse cardiac events without increasing the risk of bleeding complications. ${ }^{16}$ To our knowledge, this is the first case report of bile duct obstruction and concomitant proximal SBO that was relieved with prompt surgical intervention (ie, evacuation of intramural blood clots). Usually, the cause is bleeding from the jejunojejunostomy anastomosis and may require reoperation to achieve hemostasis or to revise the anastomotic site.

\section{References:}

1. Roux C. De la gastroenterostomie. Revue de chirurgie. 1893; 13:402-403.

2. Lewis CE, Jensen C, Tejirian T, Dutson E, Mehran A. Early jejunojejunostomy obstruction after laparoscopic gastric bypass: case series and treatment algorithm. Surg Obes Relat Dis. 2009; 5(2):203-207.

3. Ohi R, Yaoita S, Kamiyama T, Ibrahim M, Hayashi Y, Chiba T. Surgical treatment of congenital dilatation of the bile duct with special reference to late complications after total excisional operation. J Pediatr Surg. 1990;25:613-617.

4. Yamataka A, Ohshiro K, Okada Y, et al. Complications after cyst excision with hepaticoenterostomy for choledochal cysts and their surgical management in children versus adults. J Pediatr Surg. 1997;32:1097-1102.

5. Smith R. Hepaticojejunostomy: choledochojejunostomy. A method of intrajejunal anastomosis. Brit J Surg. 1964;51(3): 183-186.

6. Hughes CE, Conn J, Sherman JO. Intramural hematoma of the gastrointestinal tract. Am J Surg. 1977;133:276-279. 
7. Abbas MA, Collins JM, Olden KW, et al. Spontaneous intramural small-bowel hematoma: clinical presentation and longterm outcome. Arch Surg. 2002;137:306-310.

8. Zangan SM, Yousefzedah DK. Occlusive intramural hematoma. Pediatr Radiol. 2004;34:564-566.

9. Fischer MG. Bleeding from stapler anastomosis. Am J Surg. 1976;131:745-747.

10. Wassner JD, Yohai E, Heimlich HJ. Complications associated with the use of gastrointestinal stapling devices. Surgery. 1977; 82:395-399.

11. Petrassi A, Roncone A, Formisani P, Iannello A. Risultati dello multicentrico (A.C.O.I. Stapler Study Group) su 420 anastomosi esofago-digiunali e 544 colorettali (in Italian with English abstract). Ann Ital Chir. 1994;65:49-58.
12. Felsher J, Brodsky J, Brody F. Small bowel obstruction after laparoscopic Roux-en-Y gastric bypass. Surgery. 2003;134(3):501505 .

13. Birns MT, Katon RM, Keller F. Intramural hematoma of the small intestine presenting with major upper gastrointestinal hemorrhage. Gastroenterology. 1979;77:1094-1100.

14. Eiland M, Han SY, Hicks GM. Intramural hemorrhage of the small intestine. JAMA. 1978;239:139-142.

15. Husain S, Ahmed AR, Johnson J, Boss T, O'Malley W. Small bowel obstruction after laparoscopic Roux-en-Y gastric bypass. Etiology, diagnosis, and management. Arch Surg. 2007;142(10):988-993.

16. Oscarsson A, Gupta A, Fredrikson M, et al. To continue or discontinue aspirin in the perioperative period: a randomized, controlled clinical trial. Br. J. Anaesth 2010;104(3):305-312. 